\title{
An improved capacitive divider probe for plasma potential measurements in the Phaedrus tokamak
}

\author{
E. Y. Wang, a) D. Diebold, P. Nonn, J. Pew, W. Q. Li, , ${ }^{\text {a) }}$ P. Probert, R. Breun, R. Majeski, \\ S. L. Yan, ${ }^{\text {,) }}$ and N. Hershkowitz \\ Department of Nuclear Engineering and Engineering Physics, University of Wisconsin-Madison, \\ Wisconsin 53706
}

(Received 20 December 1990; accepted for publication 7 February 1991)

\begin{abstract}
High bandwidth capacitive probes are useful tools for potentials measurements in the tokamak scrape off layer. An improved capacitive divider probe design with very high frequency bandwidth is shown. The gain of this system is between 0 and $-8 \mathrm{~dB}$ for frequencies from $0.1 \mathrm{~Hz}$ to more than $100 \mathrm{MHz}$, with the $-3 \mathrm{~dB}$ point at approximately 40 MHz. The probe structure, circuits, performance, and experimental results from the Phaedrus-T tokamak are given.
\end{abstract}

\section{INTRODUCTION}

rf sheath physics near the Faraday shield and limiter of tokamaks can be studied with a combination of Langmuir and rf probes. For example, TEXTOR ${ }^{1}$ and ASDEX ${ }^{2}$ have used probes to measure modifications in the edge electric fields during ICRF. Probes on JET ${ }^{3}$ also indicate that the edge plasma is modified during ICRF.

High-impedance capacitive probe techniques may be more suitable for determining potential than emissive probes or particle beams in strong ICRF conditions, such as the edge of tokamak plasma in the presence of high power ICRF.' A capacitive probe can successfully track the floating potential without significantly loading the plasma provided that the impedance through the probe between the plasma and ground is large at all frequencies of interest, and provided that sheath effects may be neglected. ${ }^{4}$ In addition it has been shown that a secondary electron emission-capacitive probe can determine the plasma potential when $T_{e}>50 \mathrm{eV} .^{5}$

Phaedrus-T is an "ISX class" tokamak that began operation in the Fall of 1989. It has a major radius of $0.93 \mathrm{~m}$. The minor radius is set to $0.26 \mathrm{~m}$ by a carbon limiter. The emphasis of the Phaedrus-T tokamak program is high power ICRF studies. The ICRF frequency can be operated in the range of 13 to $18 \mathrm{MHz}$.

The active capacitive divider described in this paper is a significant improvement on older high-impedance capacitive probe techniques, enabling a very wide bandwidth from $0.1 \mathrm{~Hz}$ to $40 \mathrm{MHz}$. Experinental results from Phaedrus-T are presented.

\section{PROBE CONSTRUCTION}

The probe consists of a metallic electrode that is connected to the inner conductor of a coaxial line. The electrode, as well as the coaxial line, is then surrounded by a glass or ceramic tube. When the probe is inserted into a plasma, the plasma charges the outside surface of the glass or ceramic tube to the floating potential, and the detecting electrode couples capacitively to this potential. When the plasma electron temperature $T_{e}$ is greater than $50 \mathrm{eV}$, glass has an effective electron emission coefficient of approximately 1.0 or greater. Under such a condition, a secondary emission capacitive probe can be used determine the plasma potential. ${ }^{5}$

The divider circuit described in this article is designed to measure very rapid voltage transients accurately. The emphasis in this work is directed toward obtaining improved frequency bandwidth of $\mathrm{rf}$ plasma and/or floating potential fuctuations by using a cylindrical capacitor that is connected as close as possible to the probe electrode. A great advantage of this divider is the elimination of connecting circuits between the two arms of the voltage divider. Specifically, most of the undesirable effects of resonances caused by the inductances between the two arms and the buffer/line driver which is placed immediately af ter the probe are eliminated. In Fig. 1 we show a longitudinal section of the probe, which is symmetric around the housing axis. The attenuation factor of the capacitive divider is in the ratio of $C_{2} / C_{1}$ (when $C_{2} \gg C_{1}$ ). The capacitance $C_{2}$ is given by the cylindrical capacitor which is connected to the probe electrode directly. For our probe we choose a $C_{2}$ approximately equal to $2.5 \mathrm{nF}$. The tip capacitance $C_{1}$ is the capacitance between the electrode, which is inserted into the ceramic or glass tube, and the surface of that tube which is exposed to the plasma. A typical value for $C_{1}$ is $1-2 \mathrm{pF}$.

\section{EQUIVALENT CIRCUITS OF CAPACITIVE DIVIDER AND TYPICAL PERFORMANCE}

If the round trip time of an e.m. signal along the capacitor $C_{2}$ is negligible, the probe may be considered equivalent to the circuit of Fig. 2. The lower limit of frequency that can be measured depends on the time constant $C_{2}\left(R_{w}+R_{0}\right)$, where $R_{w}$ and $R_{0}$ are the center wire and buffer/line driver input resistance, which may be regulated to a large extent through a suitable choice of $R_{0}$. A typical

\footnotetext{
a) Southwestern Institute of Physics, Leshan, Sichuan, P.R. China.

${ }^{b}$ Shanghai Microwave Technology Institute, Shanghai, P.R. China.
} 


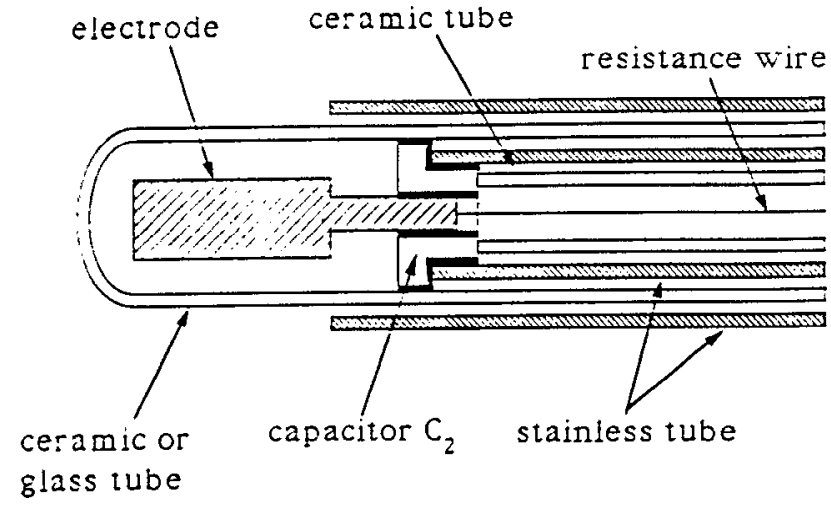

FIG. 1. Schematic drawing of the capacitive divider probe.

value of $R_{0}$ is $10^{9}-10^{10} \Omega$. On the other hand, in view of the small value of $C_{1}$, the upper limit is essentially given by the stray inductances in the low-voltage arm of the circuit rather than the time constant $R_{s} C_{1}$, where $R_{s}$ is the sheath resistance of the probe. The internal $L_{2}$ of $C_{2}$ introduces a resonance frequency $\left(L_{2} C_{2}\right)^{1 / 2}$ which is high enough so as not to be limiting, while the remaining inductance $L_{s}$ along the probe shaft is that of the center wire. If it is made large enough, the resistance of the center wire can eliminate the resonance at $\left(L_{s} C_{0}\right)^{1 / 2}$ (when $C_{2} \gg C_{0}$ ) and make the frequency response of the probe essentially flat. To accomplish this, the typical wire resistance $R_{s}$ is chosen to be $100-200 \Omega$.

A more convenient method of constructing a capacitive divider probe is to place the dividing capacitor $C_{2}$ near the buffer/line driver (rather than near the probe tip capacitance $C_{1}$ ) and to use regular, low resistance wire for the center wire (rather than high resistance wire). The equivalent circuit of such a probe is the same as Fig. 2, but with $L_{2}$ and $C_{2}$ placed near $C_{0}$ and $R_{0}$ rather than $C_{1}$ and with $R_{u}$ eliminated. When we constructed such a circuit, we found a very large resonance at approximately $15 \mathrm{MHz}$ ( $V_{\text {out }} / V_{\text {in }}$ was about 30 times greater at this resonance frequency than it was at nonresonance frequencies). We believe that $L_{s}$ in this case was about $50 \mathrm{nH}$ (the probe shaft was about $1 \mathrm{~m}$ in length) and that the resonance was caused by $V_{\text {in }}, L_{S}$, and $C_{2}$ being in series $\left(f_{\text {resonance }}=1 / 2 \pi\right.$ $\sqrt{L_{5} C_{2}}=15 \mathrm{MHz}$ ). When the dividing capacitor was placed near the probe tip, the lowest frequency large reso-

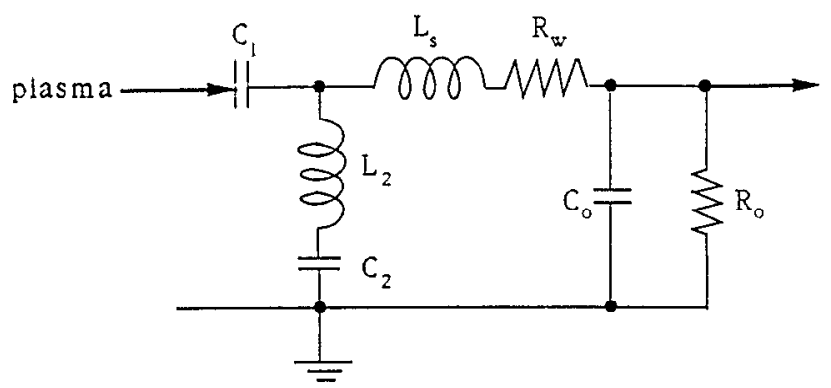

FIG. 2. Schematic circuit equivalent for probe with tip immersed in plasma.

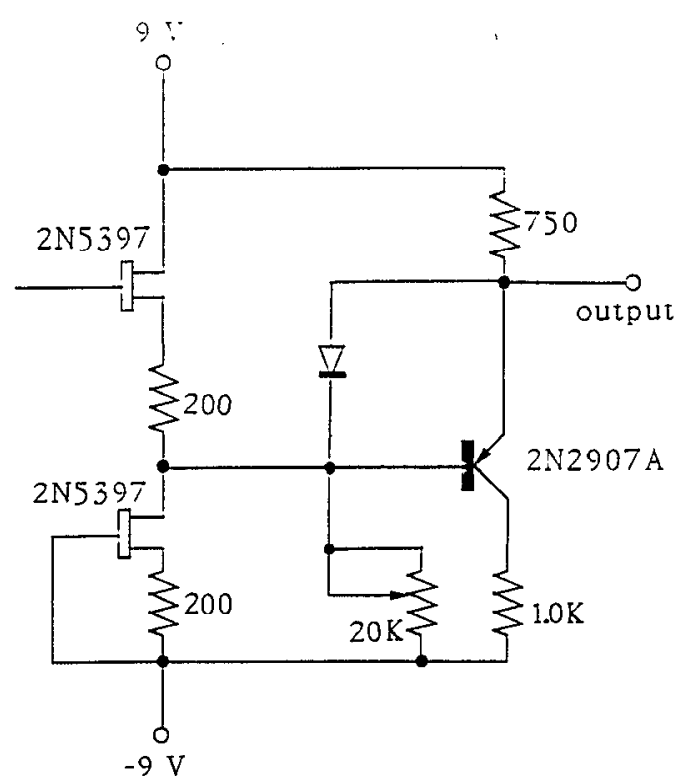

FIG. 3. Follower circuit.

nance that we measured was at approximately $70 \mathrm{MHz}$. We believe that this resonance was caused by $V_{\text {in }}, L_{s}$, and $C_{0}$ (which was about $100 \mathrm{pF}$ ) being in series $\left(f_{\text {resonance }}=1 / 2 \pi \sqrt{L_{s} C_{0}}=70 \mathrm{MHz}\right)$. It was not until we replaced the center wire high resistance wire that we found no large resonances for frequencies below $100 \mathrm{MHz}$.

It is not enough for the probe alone to be have good frequency response. The buffer/line driver must have a high input resistance for adequate low frequency response and the buffer/line driver must have high frequency response. This is accomplished by the circuit shown schematically in Fig. 3. This circuit can be constructed in small box and placed immediately after the probe. This buffer/ line driver circuit is capable of directly driving a $50 \Omega$ line.

Frequency response measurements and voltage output calibration were performed by covering the probe with alu-

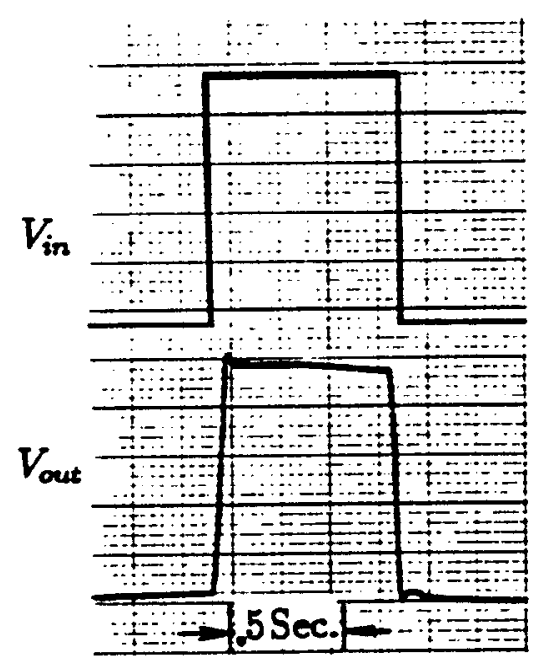

FIG. 4. Waveform of $V_{\text {in }}$ and $V_{\text {out }}$ showing a low-frequency response. 


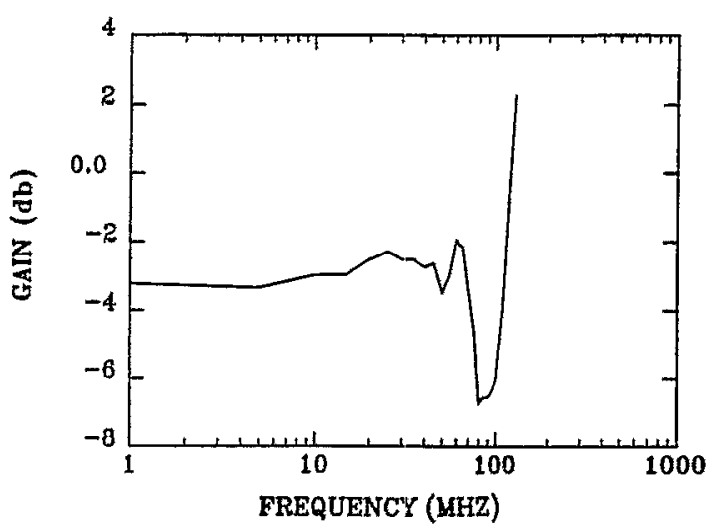

FIG. 5. The frequency response of capacitive divider probe.

minum foil or inserting the probe into mercury. The lowfrequency response of the probe was measured by applying a stcp of square signal $V_{\text {in }}$ to aluminum foil. Both $V_{\text {in }}$ and the corresponding output signal $V_{\text {out }}$ from the follower are shown in Fig. 4. The time constant is approximately $10 \mathrm{~s}$. The response of the probe to frequencies of up to more than $100 \mathrm{MHz}$ was measured. As shown in Fig. 5, the variation in voltage was less than $15 \%$ for frequencies up to $70 \mathrm{MHz}$ and less than $35 \%$ for frequencies up to 120 MHz. Figure 6 shows that frequency response of the probe/buffer system can be up to $50 \mathrm{MHz}$. This frequency response satisfies the requirements of Phaedrus-T tokamak ICRF experiments.

\section{POTENTIAL MEASUREMENTS IN THE SCRAPE OFF LAYER OF THE PHAEDRUS-T TOKAMAK}

All probe buffer/line driver and digital data storage electronics are grounded at a point on Phaedrus- $T$ 's vacuum vessel close to the probe. This eliminates the need for isolation amplifiers and the associated loss of frequency response. It is very important to reduce ground loop noise because the capacitive divider has attenuation of $10^{3}-10^{4}$. The follower output signals are approximately tens of $\mathrm{mV}$.

Experiments on several confinement devices have shown an increase in the impurity concentration in the

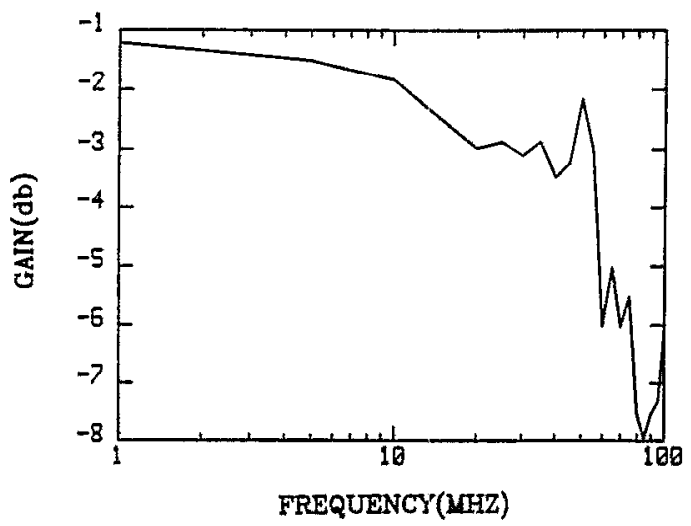

FIG. 6. The frequency response of capacitive divider probe/buffer system.

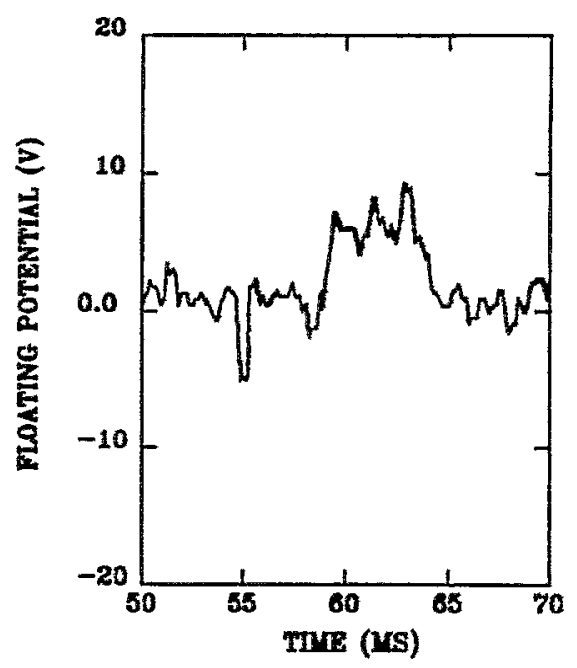

FIG. 7. A typical floating potential measured under the ICRF discharge in the Phaedrus tokamak.

plasma during ICRF heating. Specifically, the Faraday shield of the activated antenna has been identified as a local impurity source. However, the processes taking place at the antenna that cause impurity generation have not been identified. One area that needs to be addressed in understanding the impurity generation is the role of the plasma sheath that forms on the material boundaries in contact with the plasma. The magnitude and form of the plasma potential in front of the antenna must be known in order to determine the effect of this potential on the energy of the ions hitting the shield. Figure 7 shows representative floating potential data measured in the presence of an ICRF discharge in the Phaedrus-T tokamak. The potential peak is associate with the application of ICRF. The potential which oscillates at the of frequency can be measured by the capacitive probe because of the high frequency bandwidth of the probe and probe circuit. Figure 8 gives high frequency signal spectrum. Note the rf at 2 and $3 \omega_{c i}$ which

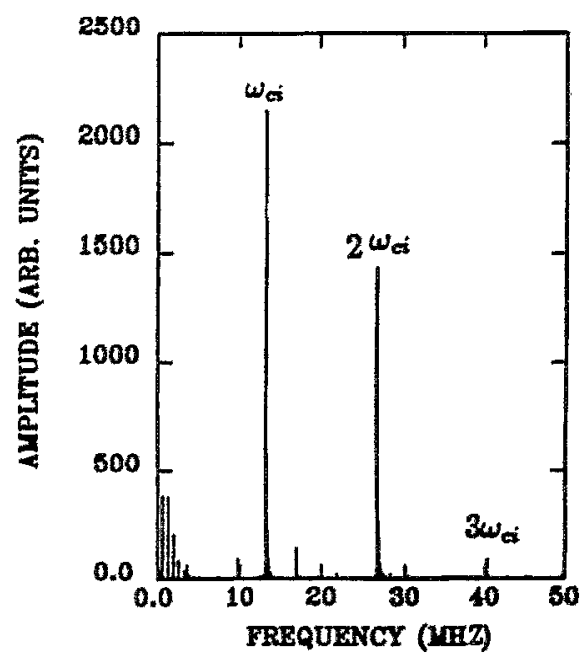

FIG. 8. Frequency spectrum of the capacitive probe signal. 


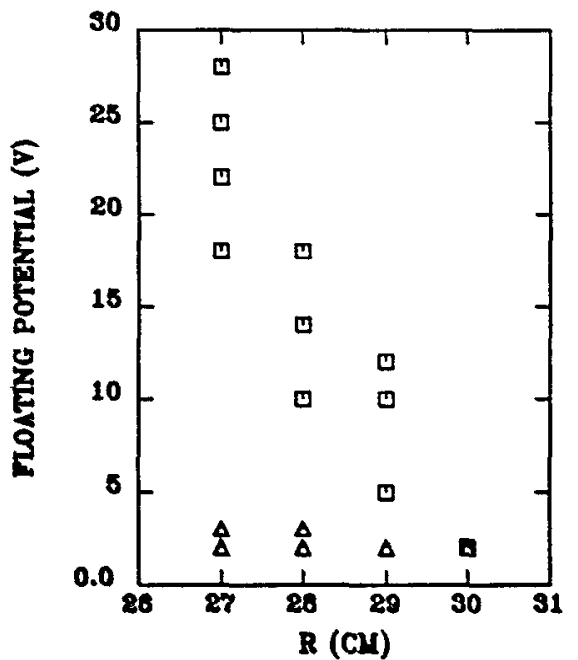

FIG. 9. Radial potential profile for an ohmic(triangle points) and ICRF (square points) heated discharge in the Phaedrus-T tokamak.

indicates the appearance of nonlinear effects depends on the presence of plasma. The effects of the electron temperature and the rf field on the potential formation in front of the antenna must also be determined before these data can be interpreted.

The recent interest in measuring radial electric fields on tokamaks is due to expcrimental as well as theoretical indications that dc electric fields play a crucial role in confinement physics and especially in attaining the $\mathrm{H}$ mode.
The plasma space potential $\left(V_{p}\right)$ can be calculated from the measured floating potential $\left(V_{f}\right)$ and electron temperature profiles $T_{e}(r)$ by using the relation $V_{p}=V_{f}-V_{s}$, where $V_{s}$ is the sheath potential at the edge of the plasma. The sheath potential can be calculated using the expression $^{6}$

$$
V_{s}=\left(T_{e} / 2\right) \ln \left[4 \pi\left(m_{e} / m_{i}\right)(1-\delta)^{-2}\right],
$$

where $\delta$ is the secondary electron emission yield, $m_{e}$ and $m_{i}$ are electron and ion mass, respectively, and it has been assumed that $T_{e}=T_{i}$. Figure 9 shows the radial profile of $V_{f}$ for an ohmic and ICRF heated discharge in the Phaedrus tokamak. Modifications in the edge electric fields during ICRF have been found.

\section{ACKNOWLEDGMENT}

This work was supported by U.S. DOE Grant No. DE-FG02-88ER53264.

${ }^{1}$ R. Van Nieuwenhove, F. Durodie', R. Koch, and G. Van Oost, Fus. Eng. Design 12, 203 (1990).

${ }^{2} \mathrm{~J}$. M. Noterdaeme and the ASDEX Team, 14th European Conf. on Controlled Fusion and Plasma Physics, Madrid, Europhysics Conference Abstracts (European Physical Society, 1987), Vol. 11D, p. 678.

${ }^{3}$ J. A. Tagle, M. Laux, S. Clement, S. K. Erents, H. Brinkschulte, M. Rures, and L. De Kock, Fus. Eng. Design 12, 217 (1990).

${ }^{4}$ S. E. Savas and K. G. Donohoe, Rev. Sci. Instrum. 60, 3391 (1989).

${ }^{5}$ E. Y. Wang, N. Hershkowitz, D. Diebold, T. Intrator, R. Majeski, H. Persing, G. Severn, B. Nelson, and Y. J. Wen, J. Appl. Phys. 61, 4786 (1987).

${ }^{6}$ J. A. Tagle, M. Laux, S. Clement, S. K. Erents, H. Brinkschulte, M. Bures, and L. De Kock, Fus. Eng. Design 12, 217 (1990). 